\title{
Medium Magnetizations for Longitudinal High-Density Digital Recordings
}

\author{
B. K. Middleton, Member, IEEE, M. M. Aziz, Member, IEEE, and D. E. Speliotis, Life Fellow, IEEE
}

\begin{abstract}
This paper reports on the influences of magnetic media remanent magnetizations on the output levels in digital magnetic tape and disk recording. While at low densities a high magnetization is required for a high output, a lower magnetization is required at high densities. However, for particular applications, an output near to the maximum can be obtained from a wide range of remanent magnetization values, which suggests possible uses for media with diverse properties.
\end{abstract}

Index Terms-Magnetic recording, remanent magnetization.

\section{INTRODUCTION}

$\mathbf{T}$ HERE has been much discussion over the years on the influence of the magnetic hysteresis properties of recording media on their digital recording performances. Much of this discussion has concentrated on the influence of media thickness and coercivity on recorded transition widths, which need to be kept small to ensure large and narrow replayed pulses. It has led to the universally accepted requirement for high-density recording media that they be thin and of high coercivity. When it comes to the influence of the remanent magnetization on high-density operation, there needs to be further discussion, and this is the purpose of this paper. The relevant theoretical background is provided and predictions are made of the magnitudes of the remanent magnetizations needed for high outputs at high recording densities. Two media thicknesses are considered which correspond to thin tapes and thin disks.

\section{THEORY}

\section{A. Output Voltages}

The output voltage amplitude $e(0)$ generated in a Karlqvist type head by an alternating sequence of arctangent shaped transitions has been calculated by many authors. Here, expression (40) of Middleton et al. [1], with some changes to symbols, has been manipulated to represent the rolloff curve as

$$
\begin{aligned}
e(0)= & \left(\frac{2}{\pi}\right) \mu_{o} v w M_{r} n \eta g \\
& \cdot \ln \left(\frac{\tanh \left(\frac{\pi(a+d+\delta)}{\lambda}\right)}{\tanh \left(\frac{\pi(a+d)}{\lambda}\right)}\right) \frac{\sin \left(\frac{\pi g}{\lambda}\right)}{\left(\frac{\pi g}{\lambda}\right)}(a+d)>2 g
\end{aligned}
$$

Manuscript received January 29, 2002; revised July 25, 2002.

B. K. Middleton is with the Department of Computer Science, University of Manchester, Manchester M13 9PL, U.K. (e-mail: B.Middleton@cs.man.ac.uk).

M. M. Aziz is with the School of Engineering and Computer Science, University of Exeter, Exeter EX4 4QF, U.K. (e-mail: M.M.Aziz@exeter.ac.uk).

D. E. Speliotis is with the Advanced Development Corporation, Lexington, MA 02420 USA (e-mail: dspeliotis@ade.com).

Digital Object Identifier 10.1109/TMAG.2002.806350 where $a$ is the arctangent transition width parameter, $M_{r}$ is the remanent magnetization, $d$ is the head to medium spacing, $\delta$ is the medium thickness, $g$ is the replay head gap length, $\lambda$ is the wavelength of recording, $n$ is the number of turns on the head which has efficiency $\eta, w$ is the track width, $v$ is the medium velocity, and $\mu_{o}$ is the permeability of free space.

The transition widths have, likewise, been predicted by many authors, but here the calculations of Middleton [2], originally carried out for very thin media, have been repeated with the restriction of the thin film approximation lifted and the result for a write limited transitions is

$$
\begin{aligned}
& a=-\frac{\delta}{4}+\frac{M_{r} y}{\pi \chi Q H_{c}} \\
&+\sqrt{\left(\frac{\delta}{4}-\frac{M_{r} y}{\pi \chi Q H_{c}}\right)^{2}+\frac{M_{r} \delta y(1+\chi)}{\pi \chi Q H_{c}}} \\
& y=d+\frac{\delta}{2}
\end{aligned}
$$

where $H_{c}$ is the medium coercivity, $\chi$ is the slope of the hysteresis loop at the coercive field which is often given as $\chi=$ $\left(M_{r} / H_{c}\right) /\left(1-S^{*}\right)$ [3], and $Q$ is a factor introduced by Williams and Comstock [4] to account for the influence of head gap length and record current amplitude on head field gradient [3], [4]. $Q$ has values less than or equal to unity, and for a narrow gap head receiving near to optimum record currents, as assumed here, the value is unity. This value is used in all the following numerical calculations.

Substituting for $\chi$ in (2a) leads to

$$
\begin{aligned}
a= & -\frac{\delta}{4}+\frac{y\left(1-S^{*}\right)}{\pi Q} \\
& +\sqrt{\left(\frac{\delta}{4}-\frac{y\left(1-S^{*}\right)}{\pi Q}\right)^{2}+\frac{M_{r} \delta y\left(1+\frac{H_{c}}{M_{r}}\left(1-S^{*}\right)\right)}{\pi Q H_{c}}} \\
y= & d+\frac{D}{2} .
\end{aligned}
$$

For rectangular loops, (2a) and (2b) become

$$
\begin{aligned}
& a=-\frac{\delta}{4}+\sqrt{\left(\frac{\delta}{4}\right)^{2}+\frac{M_{r} \delta y}{\pi Q H_{c}}} \\
& \chi \rightarrow \infty, \quad S^{*} \rightarrow 1 .
\end{aligned}
$$

Using (1), with transition width given by (2b), the rolloff curves for three media with magnetization values of 300,150 , and $100 \mathrm{kA} / \mathrm{m}(\mathrm{emu} / \mathrm{cc})$ and $S^{*}=0.8$ are shown in Fig. 1. The medium thickness is assumed to be $100 \mathrm{~nm}$ corresponding to a thin tape. At low densities, the medium with the higher magnetization has the highest output, while at very high densities, it is 


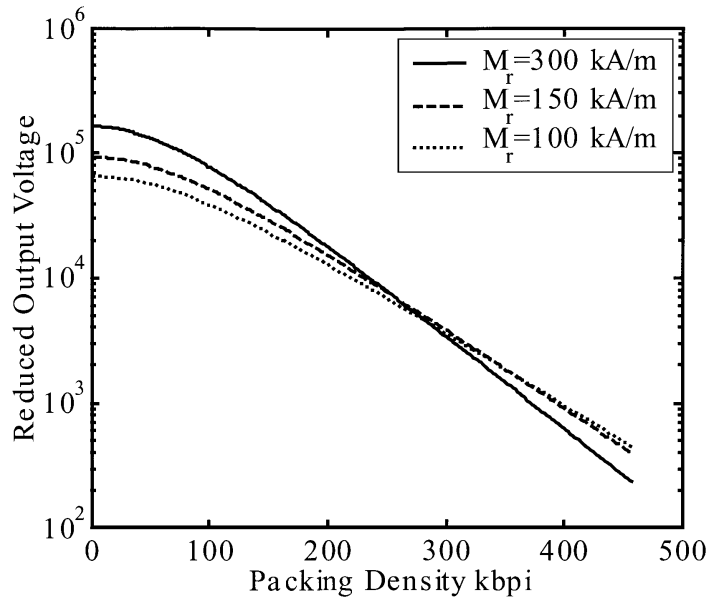

Fig. 1. Reduced output voltage $e(0) /\left((2 / \pi) \mu_{0} v w n \eta g\right)$, from (1) and (2b), as a function of packing density using $M_{r}=300,150$, and $100 \mathrm{kA} / \mathrm{m}(\mathrm{emu} / \mathrm{cc})$, $H_{c}=160 \mathrm{kA} / \mathrm{m}, S^{*}=0.8, d=40 \mathrm{~nm}, \delta=100 \mathrm{~nm}, g=1 \mathrm{~nm}$ (i.e., a narrow gap head).

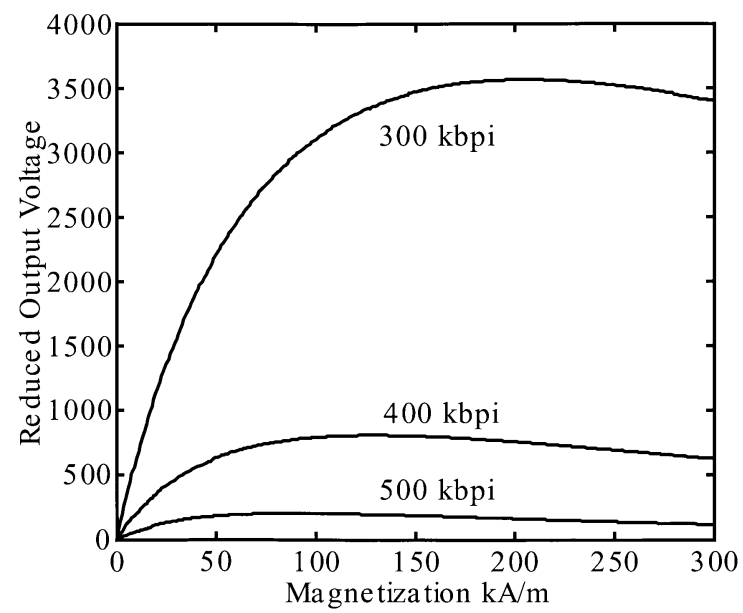

Fig. 2. Reduced output voltage as a function of $M_{r}$ from (3) and (2b), for the same parameters as used in Fig. 1.

the medium with the lowest magnetization which produces the highest output.

At high densities, (1) can be approximated to

$$
\begin{aligned}
e(0)= & \left(\frac{4}{\pi}\right) \mu_{o} v w M_{r} n \eta g \\
& \cdot e^{-2 \pi(a+d) / \lambda}\left(1-e^{-2 \pi \delta / \lambda}\right) \frac{\sin \left(\frac{\pi g}{\lambda}\right)}{\left(\frac{\pi g}{\lambda}\right)} \\
\lambda< & (a+d)
\end{aligned}
$$

and from (3) and (2b), the variation of output amplitude as a function of $M_{r}$ is shown in Fig. 2 again for the thicker tape medium. This reveals that while there is a value of magnetization which maximizes the output, there is a wide range of magnetization values that produce outputs near to the maximum. The existence of a maximum comes about because (3) is made up of a linear term involving magnetization multiplied by an exponential term which decreases with increasing magnetization

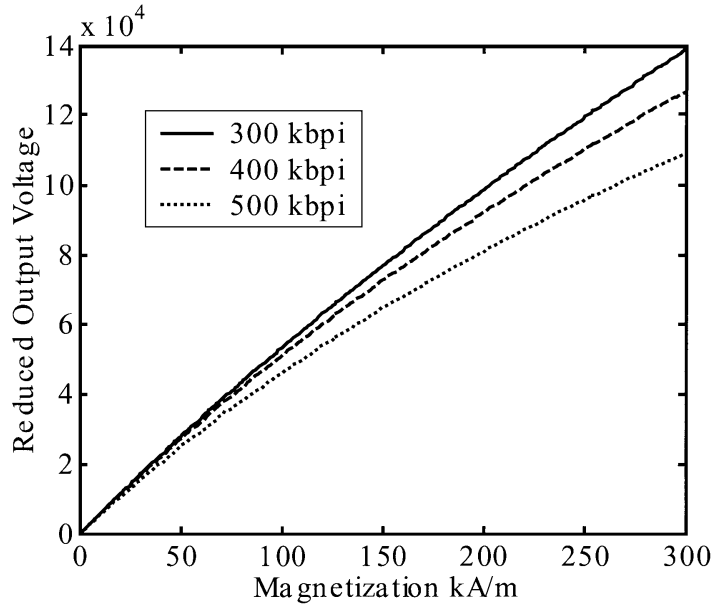

Fig. 3. Reduced output voltage as a function of $M_{r}$ from (3) and (2b) for thin-film disk media with $H_{c}=239 \mathrm{kA} / \mathrm{m}(3000 \mathrm{Oe}), \delta=20 \mathrm{~nm}$, and $(d+$ $\delta / 2)=25.4 \mathrm{~nm}$.

with the result that there is an optimum value [5]. The values of magnetization used in the formation of Fig. 2 are approximately those available in media today and show that current magnetizations are near to the optimum. However, when values closer to those of current thin-film disk media are used, Fig. 3 shows that they are way below the optimum.

\section{B. Optimum Magnetization at High Densities}

Maximization of the output (3) with respect to $M_{r}$ at high densities leads to the condition for optimum magnetization $M_{r}^{\text {opt }}$ at wavelength $\lambda_{m}$ of

$$
\frac{d a}{d M_{r}}=\frac{\lambda_{m}}{2 \pi M_{r}^{\text {opt }}}
$$

Substitution of (2a) into (4) and evaluation leads to

$$
\begin{aligned}
& \lambda_{m}= \frac{2 M_{r}^{\mathrm{opt}} y}{\chi Q H_{c}} \\
&+\frac{\pi M_{r}^{\mathrm{opt}}\left\{2\left(\frac{\delta}{4}-\frac{M_{r}^{\mathrm{opt}} \delta}{\pi \chi Q H_{c}}\right)\left(\frac{-y}{\pi \chi Q H_{c}}\right)+\frac{\delta y(1+\chi)}{\pi \chi Q H_{c}}\right\}}{\sqrt{\left(\frac{\delta}{4}-\frac{M_{r}^{\mathrm{opt}} y}{\pi \chi Q H_{c}}\right)^{2}+\frac{M_{r}^{\mathrm{opt}} \delta y\left(1+\frac{1}{\chi}\right)}{\pi Q H_{c}}}}
\end{aligned}
$$

which, with substitution for $\chi$, becomes

$$
\begin{aligned}
& \lambda_{m}=\frac{2 y\left(1-S^{*}\right)}{Q} \\
& +\frac{\frac{y\left(1-S^{*}\right)}{Q}\left\{\frac{M_{r}^{\mathrm{opt}}}{H_{c}\left(1-S^{*}\right)}\left(1+\frac{H_{c}}{M_{r}^{\mathrm{opt}}}\left(1-S^{*}\right)\right)-\frac{\delta}{2}\left(1-\frac{4\left(1-S^{*}\right)}{\pi Q}\right)\right\}}{\sqrt{\left(\frac{\delta}{4}-\frac{y\left(1-S^{*}\right)}{\pi Q}\right)^{2}+\frac{M_{r}^{\mathrm{opt}} \delta y}{\pi Q H_{c}}\left(1+\frac{H_{c}}{M_{r}^{\mathrm{opt}}}\left(1-S^{*}\right)\right)}}
\end{aligned}
$$

For rectangular hysteresis loops, (5a) and (5b) reduce to

$$
\lambda_{m}=\frac{\frac{M_{r}^{\mathrm{opt}} \delta y}{Q H_{c}}}{\sqrt{\left(\frac{\delta}{4}\right)^{2}+\frac{M_{r}^{\mathrm{opt}} \delta y}{\pi Q H_{c}}}} \quad \chi \rightarrow \infty \quad S^{*} \rightarrow 1 .
$$




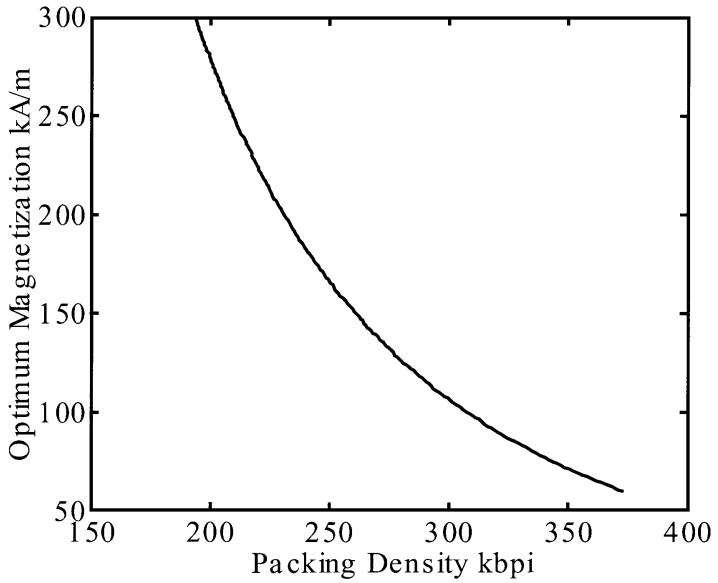

Fig. 4. $\quad M_{r}^{\text {opt }}$ as a function of packing density. Parameters are as for Fig. 1.

Equations (5a)-(5c) show that as wavelengths decrease, so do the values of $M_{r}^{\text {opt }}$ [5]. Inversion of (5a) and (5b) is only feasible numerically, but inversion of $(5 \mathrm{c})$ is straightforward and leads to

$$
\begin{aligned}
M_{r}^{\mathrm{opt}} & =\frac{\lambda_{m}^{2} Q H_{c}}{2 \pi \delta y}\left\{1+\sqrt{1+\left(\frac{\pi \delta}{2 \lambda_{m}}\right)^{2}}\right\} \\
& =\frac{2 Q H_{c}}{\pi \delta y D_{m}^{2}}\left\{1+\sqrt{1+\left(\frac{\pi \delta D_{m}}{4}\right)^{2}}\right\} \chi \rightarrow \infty
\end{aligned}
$$

where $D_{m}=2 / \lambda_{m}$ is the packing density corresponding to the wavelength $\lambda_{m}$. Equation (6) clearly shows that the optimum values of magnetization reduce as packing densities increase and this is confirmed in Fig. 4, which has been determined using (5b) and the parameters of the thicker media.

The essence of this analysis is not changed by substituting a shielded magnetoresistive head for the inductive head since the output waveforms bear significant similarities [6]. This is so provided the heads remain in their linear regions, otherwise nonlinear effects on replay will need to be considered. However, with a noisy medium, the power signal-to-noise ratio (SNR) at the replay head will be important. While noise sources which relate to the granularity of the media may be diverse in their origins, they invariably generate powers proportional to $M_{r}^{2}$ [3], [7]. Thus, the power SNR, on discarding spacing, thickness, and gap losses in (3) is

$$
\mathrm{SNR} \propto e^{-2 \pi a / \lambda}
$$

which decreases as $M_{r}$ increases, and so a low magnetization is the requirement. However, noise sources from other than the medium mean that an optimum magnetization occurs.

\section{CONCLUSION}

It has been shown that in media of thickness around $100 \mathrm{~nm}$ as recording densities increase, low values of magnetization are needed to maximize outputs. However, a wide range of magnetization values are capable of producing outputs near to the maxima leading to the possible use of a range of media for high-density digital recording. When thin-film disk media are considered, the current magnetization values are found to be substantially less than the optima.

\section{REFERENCES}

[1] B. K. Middleton, C. D. Wright, S. R. Cumpson, and J. J. Miles, "Output waveforms in the replay process in digital magnetic recording," IEEE Trans. Magn., vol. 31, pp. 2365-2379, May 1995.

[2] B. K. Middleton, "The recording and reproducing processes," in Magnetic Recording Technology, C. D. Mee and E. D. Daniel, Eds. New York: McGraw-Hill, 1996, ch. 2, pp. 2.1-2.72.

[3] H. N. Bertram, Theory of Magnetic Recording. Cambridge, U.K.: Cambridge Univ. Press, 1994.

[4] M. L. Williams and R. L. Comstock, "An analytical model of the write process in digital magnetic recording," in Proc. 17th Annu. API Conf., vol. 5, 1971, pp. 738-742.

[5] D.E. Speliotis, private communication.

[6] B. K. Middleton, "Modeling the digital magnetic recording behavior of shielded magneto-resistive replay heads with displaced elements," Rad. Elec. Eng., vol. 50, pp. 419-425, Aug. 1980.

[7] M. M. Aziz, B. K. Middleton, and J. J. Miles, "Autocorrelation analysis of particle magnetization in erased particulate media," IEEE Trans. Magn., vol. 38, pp. 279-287, Jan. 2002. 